\title{
Research on Software Resources Encapsulation Technology Based on Cloud Manufacturing Service
}

\author{
Dongfang $\mathrm{Hu}^{1, \mathrm{a}^{*}}$, Yuanhao $\mathrm{Ji}^{1, \mathrm{~b}}$, Yan Zhao ${ }^{2, \mathrm{c}}$ \\ ${ }^{1}$ School of Mechantronics Engineering, Henan University of Science and Technology, Luoyang, \\ 471003, China \\ 'LandGlass Technology Co., Ltd, Luoyang, 471003, China \\ ahdf@haust.edu.cn, bjustin910919@163.com, chlxc-2009@163.com
}

Keywords: Cloud Manufacturing Service; Software Resources; Encapsulation Technology; Web Service

\begin{abstract}
The encapsulation technology of manufacturing resources for software is studied, which can efficiently share software resources under the environment of cloud manufacturing service. We propose a general encapsulation method of cloud manufacturing resources based on Web Service. Firstly, cloud manufacturing resources are classified reasonably, and a unified model of description and interface for cloud manufacturing resources is built. Moreover, the general process of software resources encapsulation is analyzed. Finally, we put the encapsulation of the simulation analysis software ANSYS as an example to verify the feasibility of description model, interface model and encapsulation process for software resources.
\end{abstract}

\section{Introduction}

As a new service mode of manufacturing, the concept of cloud manufacturing has received more and more attention and research since it was formally put forward by the ministry of science and technology in 2010. Cloud manufacturing that is in the concept of "manufacturing as a service" have achieved the efficiently use of resources and services based on the virtual encapsulation technology [1] [2]. Description of manufacturing resources and extraction of functional interface are the key to the realization of resource encapsulation, and domestic scholars have made some research on it. Ren Lei [3] proposed a cloud manufacturing resource virtualization framework in order to realize the transformation between physical resources and logical resources, but given no specific implementation process. Li Wei-ping et al. [4] studied the resources description for manufacturing and service by the similar form of WSDL based on description method of web services. Although they have put forward a certain theoretical basis for the cloud manufacturing resource encapsulation, there is no actual packaging process.

The software resource is an important part of the cloud manufacturing resource which have the characteristics of large quantity and variety [5]. In order to realize software resources encapsulation [6], cloud manufacturing resources are classified. Then, we research on software resource description and interface extraction, and give the software resource encapsulation process. Finally, we put the simulation analysis software ANSYS as an example to verify the feasibility of model. This method can not only reduce the investment of enterprises, but also can improve the speed of product development, which is of great significance to enhance the core competitiveness of enterprises.

\section{Classification of Cloud Manufacturing Resources}

In the cloud manufacturing service system, there are kinds of manufacturing resources [7], and the reasonable classification is the first step to realize the rapid encapsulation of resources. In order to facilitate the description, we divide the cloud manufacturing resources into five categories: hardware resources, software resources, knowledge resources, information resources and other resources, as shown in Fig. 1. 


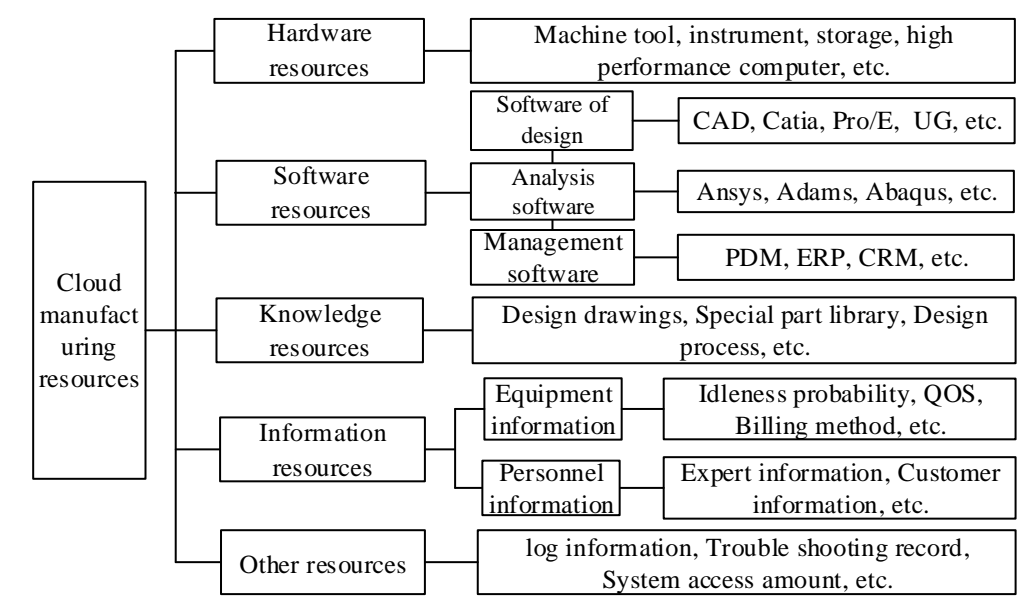

Figure 1. The classification of cloud manufacturing resources

Hardware Resources. Hardware resources mainly include various types of equipment, storage media, high performance computing and other resources for product design and manufacture, such as $\mathrm{CNC}$ machine tools, three coordinate measuring instrument, computer and high-performance computing server, etc.

Software Resources. Software resources are the collection of all the software systems needed in the whole life cycle of product collaborative design. Software resource is cloud manufacturing service of product collaborative design system is a very important class of resources, in product collaborative design process, in order to reduce the test cost of physical prototype, designers often use software for virtual design, virtual assembly and virtual simulation design tasks. According to the different functions, the software system can be subdivided into product design system, simulation analysis system and management system.

Knowledge Resources. Knowledge resources is refers to the collaborative product required in the design process and manufacture professional knowledge, including product design process of accumulation and preservation for design drawings, process design, special parts library and manage the technical document knowledge data, etc.

Information Resources. Information resources refers to the total resource information needed in the process of cloud manufacturing service system. Information resources can be divided into two major categories: manufacturing resources service information and personnel information.

Other Resources. Other resources are the collection of all resources except the above four resource types, such as system log information, trouble-shooting records, system access, etc.

\section{Description of Cloud Manufacturing Resources}

Description of cloud manufacturing resources is the key to realize the encapsulation for resources in the cloud manufacturing service system, and the process of description for resources includes resource access, resource discovery and other important information [8]. In order to standardize and simplify the description of resources, the information types of similar resources are summarized, and its generic attribute information is extracted. Then, a unified model of resource description is built to regulate the description of resources.

General Description Model of Cloud Manufacturing Resources. According to the description for different kinds of resources, the general description model of cloud manufacturing resources is defined as follows:

CMResource $=<$ BaseInfo, FunctionInfo, PerfomanceInfo, UsingInfo, StateInfo $>$.

Among them, BaseInfo is a description of the basic attributes of the resources, including the name of the resources, models, categories, version information, providers, etc. FunctionInfo is a description of the function of resources. PerfomanceInfo is a description of the resource performance attributes. UsingInfo is a description for the information that property of resources. StateInfo is a description for the state of the resource in the whole service process. 
This description model of manufacturing resource is a generic model for a certain type, the object of description for specific resource should be adjusted to the characteristics of resource classification.

Description Model of Functional Interface for Cloud Manufacturing Resources. The realization of the function for manufacturing resource can be abstracted into three processes, which are task input, task execution and result view. For example, the necessary input file of finite element analysis software is pre-processing data, and its task to be executed is the process of solution, and as a result we obtain the data file of calculation result; The necessary input file of numerical control machine is processing code, and its task to be executed is the operation for parts, and as a result we obtain finished parts. In the cloud manufacturing service system, the execution of these tasks is realized by invoking the functional interface of the corresponding resources. In order to realize the rapid encapsulation of functional interface for resources, we should extract the unified interface to describe the model according to the different types of resources, and build interface to achieve respective functions of resources.

Description model files of interface for cloud manufacturing resources are as follows:

Public interface CMResource

\{

public xml Initial(CMResBasicParamConfig config); // Resource initialization operation, such as boot parameter settings of resource, read execution file (XML), etc.

public xml setTaskInputParam(); // User need to input necessary parameter information into the process of task. Moreover, the parameter information are generally returned to the user through the system interface.

public xml executeTask(); // Perform last operation until the end of the task.

public xml getResult(); // Read task execution results which are generally described in XML format.

\}

When the user invokes the cloud manufacturing service, the primary operation is to invoke the Initial to initialize the manufacturing resource. Then, resource initialization files that is generally described as XML format are read. In the process of executing task, the relevant return parameters are set according to the requirements of task. And after the user submits the parameter through the "setTaskInputParam", the system will execute the following tasks with invoking the resources corresponding service interface. When the task is completed, user can view the results of the task through the "getResult". This description model is a generic model for classified resource, and users can make appropriate extensions to model according to the needs of the resources.

The Encapsulation Process of Cloud Manufacturing Resources Based on Web Service. The encapsulation of cloud manufacturing resource is a key to realize the service function of cloud manufacturing system [9]. The problem of heterogeneous and remote between resources has been shielded effectively, since management of resource encapsulation used XML technology based on Web Service [10]. The specific encapsulation process of cloud manufacturing resource based on Service Web is as follows:

(1) Select resource classification: The resources encapsulated are classified according to its characteristics, which is the necessary condition to use the description model of the resource classification.

(2) Description of resource properties: According to the classification of manufacturing resources, the corresponding generic description model is invoked on the cloud server. Finally, user can obtain the attribute description file WSDL based on the XML schema.

(3) Implementation of resource interface: According to the predefined interface description model, the specific function interface is implemented. According to the requirements of the functional interface, it can also extend the predefined interface description model.

(4) Publication of service: Resource providers publish the defined resource description files and implementation method of resource interface in the resources pool of cloud manufacturing service system. Then, it can provide services to other users. 


\section{The Encapsulation Example of Cloud Manufacturing Software Resources}

Simulation software is an important component of the cloud manufacturing software. ANSYS software is a typical representative of the simulation software. The process of task execution can be divided into three stages: pre-processing, solving and post-processing.

Description of Resource Attribute for ANSYS. According to the general description model of resources in the section 3, the resource attributes file of the simulation analysis software ANSYS are described as follows:

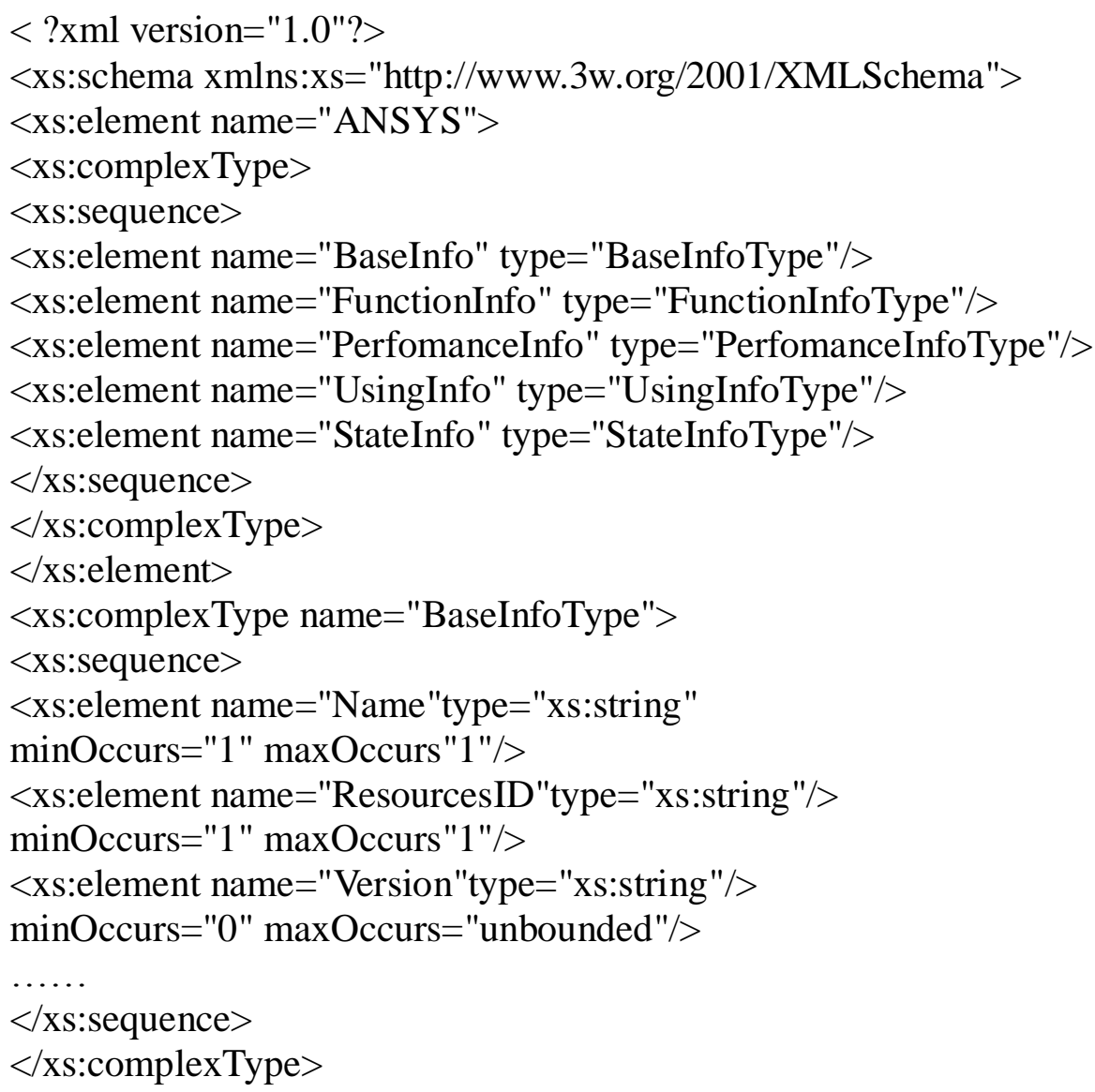

Extraction of ANSYS Function Interface. Extract and implement the function interface is the most important task of the software resource encapsulation. Three aspects are included in resources interface, namely initialization, function configuration and application configuration. Initialization function and configuration of ANSYS interface implementation methods are as follows:

public xml Initial(ANSYSConfig config);

\{

$\mathrm{xml} x$ Form=setANSYS(); // Set the return path of the simulation analysis result, and the format of the file or image.

Return xForm;

\} public xml SetUpManager(FuctionInfo, ..., ComputeMode);

\{

xml $\quad x F u n c=$ SetUpFuctionInfo(FuctionInfo); // Selection function module of ANSYS.

$\mathrm{xml} \quad \mathrm{xANSYS}=$ InitialANSYS(ANSYS); // Set invoke and return mode for function.

xml xTsf=SetUpTaskSubForm(TaskSubForm); // Set the format for submitting tasks.

$\mathrm{xml} \quad \mathrm{xCm}=\mathrm{SetUpComputeMode}$ (ComputeMode); // Set the calculation of the data.

return $\mathrm{xFunc}+\mathrm{xANSYS}+\ldots . .+\mathrm{xTsf}+\mathrm{xCm}$; \}

The interface is the key to realize the function of ANSYS to provide users with required service. 
First, the user submits the Log format of the APDL command stream file to the system through the function interface, and the system automatically turns to the task execution status. The main work of the ANSYS is set and calculate model constraints that imposed on the APDL have been set by the user. Results are generally returned in the form of data tables and analysis of cloud images, the user can view the results through the cloud service system interface online or download.

\section{Conclusion}

The ultimate goal of cloud manufacturing services is that resources can be shared efficiently. The encapsulation process of software resource is analyzed to provide some reference for other cloud manufacturing resources. The feasibility of encapsulation method of cloud manufacturing resources based on Web Service is verified through the application example of the simulation analysis software ANSYS. Resources can be shared transparently based on complete connection with cloud manufacturing service system, which can break the boundary of enterprise as well as the limitations of regional resource.

\section{Acknowledgement}

The authors gratefully acknowledge the National Nature Science Foundation (Project No. 51475142), the Key Technology R\&D Program of Henan Province (Project No. 13A520232), and the Major and Previous Pre-research Project of Henan University of Science and Technology (Project No. 2011CX016).

\section{References}

[1] B.H. LI, L. Zhang, L. Ren, et al: Computer Integrated Manufacturing Systems, Vol. 17 (2011) No.4, p.450 (In Chinese).

[2] L. Zhang, Y.L. Luo, W.H. Fan, et al: Computer Integrated Manufacturing Systems, Vol. 17 (2011) No.4, p.458 (In Chinese).

[3] L. REN, L. Zhang, Y.B. Zhang, et al: Computer Integrated Manufacturing Systems, Vol. 17 (2011) No.3, p.512 (In Chinese).

[4] W.P. Li, H.P. Lin, T. Mo, et al: Manufacturing automation, Vol. 33 (2011) No.1, p.88 (In Chinese). L.L. LI, C. YAO, Q.C. WANG: Fire Control \& Command Control, (2014) No.5, p.45 (In Chinese). L. Kong, W. Xu, J. CHA, et a1: Industrial Control and Electronics Engineering (ICICEE), (2012), p.921.

[5] S.L. Wang, W.Y. Song, L. Kang, et al: Computer Integrated Manufacturing Systems, Vol. 18 (2012) No.7, p.1396 (In Chinese).

[6] K. Chen, D.B. Wang, Y.H. Wang, et al: Manufacturing Information Engineering of China, Vol. 41 (2012) No.5, p.58 (In Chinese).

[7] W. Wang, Y.H. Zhang, X.D. Xu, et.al: Machinery Design \& Manufacture, (2012) No.8, p.260 (In Chinese).

[8] T.X. Song, C.L. Zhang, C.H. LI, et al: Computer Integrated Manufacturing Systems, Vol. 19 (2013) No.5, p.1147 (In Chinese). 